hep-th/0308173

\title{
A Positivity Theorem for Gravitational Tension in Brane Spacetimes
}

\author{
Jennie Traschen \\ Department of Physics \\ University of Massachusetts \\ Amherst, MA 01003 \\ traschen@physics.umass.edu
}

\begin{abstract}
We study transverse asymptotically flat spacetimes without horizons that arise from brane matter sources. We assume that asymptotically there is a spatial translation Killing vector that is tangent to the brane. Such spacetimes are characterized by a tension, analogous to the ADM mass, which is a gravitational charge associated with the asymptotic spatial translation Killing vector. Using spinor techniques, we prove that the purely gravitational contribution to the spacetime tension is positive definite.
\end{abstract}




\section{Introduction}

In this paper, we will study positivity properties of the gravitational mass and tension of $p$-brane spacetimes, using the spinorial methods of reference [1]. A $p$-brane spacetime is not asymptotically flat, but rather 'transverse asymptotically flat', i.e. as one approaches infinity in spatial directions transverse to the brane, the $n$-dimensional spacetime becomes $(n-p)$-dimensional flat spacetime cross a $p$-dimensional space which is 'tangent to the brane'. The tension of a p-brane spacetime [2][3] is defined in close analogy to the mass of an asymptotically flat spacetime. The gravitational contribution to the tension can be motivated as follows. A particle-like object, such as a billiard ball, has a rest mass. If this mass becomes large, then self-gravity becomes important, and there are gravitational contributions to the total mass. Now, consider an elastic band. The elastic band has a tension as well as its mass per unit length. Suppose the band is either infinitely long, or wraps around a noncontractable loop. We can ask if there is a gravitational contribution to this tension as well? The ADM mass of an asymptotically flat spacetime is defined by a boundary integral of the long range gravitational field, and can be constructed as a conserved charge associated with the asymptotic time translation Killing vector $\partial / \partial t$. In analogy with mass, one would expect the spacetime tension to be a conserved charge associated with an asymptotic spatial translation Killing vector $\partial / \partial x$ which is parallel to the brane. This notion of spacetime tension for $p$-brane spacetimes was formalized in references [2][3].

Reference [2] extended the Hamiltonian construction of gravitational charges [4] in a natural way to $p$-brane spacetimes. The procedure is roughly as follows. Let $\mathcal{H}_{t}$ be the Hamiltonian that generates the flow along a timelike vector field asymptotic to $\partial / \partial t$. Regge and Teitelboim [4][5] calculated the variation of the Hamiltonian $\delta \mathcal{H}_{t}$ under variations of the dynamical degrees of freedom. The variations must preserve the asymptotically flat boundary conditions. In order that $\delta \mathcal{H}_{t}$ vanish on solutions to the equations of motion, they found that a certain surface term must be added to the usual volume form of the Hamiltonian. This surface term is a linear combination of the ADM gravitational charges. The coefficients of the charges are the asymptotic values of the Lagrange multipliers appearing in the definition of the Hamiltonian, and asymptote to global Poincare transformations.

Now consider a $p$-brane spacetime. We assume that at transverse spatial infinity, there is a spatial Killing field $\partial / \partial x$, tangent to the brane. The covariance of the theory ensures that one can also construct a Hamiltonian $\mathcal{H}_{x}$ that generates flow along a spacelike vector field asymptotic to $\partial / \partial x$. Calculation of the variation $\delta \mathcal{H}_{x}$, with a Lagrange multiplier asymptotic to a translation in the $x$-direction, then gives a surface term proportional to the spacetime tension $\mu$. The resulting definition of $\mu$ is given in equation (18) below ${ }^{1}$.

\footnotetext{
${ }^{1}$ Reference [2] established a first law of black $p$-brane mechanics that relates variations in the tension $\delta \mu$ to variations in the other standard thermodynamic quantities. The definition of the tension $\mu$ is then obtained by integrating up the expression for $\delta \mu$ given in [2], as one does to obtain the ADM gravitational charges in the Hamiltonian approach of reference [4]. In reference [3] a
} 
In the perturbative limit the tension is given in terms of the brane stress-energy, by

$$
\mu=-\int T_{x x} d v
$$

i.e. the tension is minus the pressure of the matter stress-energy. This coincides with our intuitive definition of tension above. It is worth noting that, the tension $\mu$ is not the same as the ADM momentum in the $x$-direction, which is given in the perturbative limit by $P^{x}=\int T_{x t} d v$.

In this work we will show that the tension can be written as a contribution from the matter stress-energy $T_{a b}$ and a purely gravitational contribution. As in the case of gravitational mass, it is natural to ask if the tension is sign definite. Using techniques similiar to those of Witten and Nester in proving the positive mass theorem [1] [7], we prove that the purely gravitational contribution to $\mu$ is positive definite. The first step is to define the spacetime tension $\mu$ in terms of the long range gravitational field and a spinor field, and show that the spinor definition of $\mu$ agrees with the previously given definition. If the spacetime is static throughout the expression for

$\mu$ simplifies. Further, we see that if a static spacetime has a local boost invariance in the $x-t$ directions, then then the mass per unit length minus the tension just depends on stress-energy terms. Horizons add a significant technical complication. Since the proof uses Stokes Theorem, a horizon introduces an additonal boundary term, which one would like to show is geometrical, or nonnegative. In the case of the mass, reference [8] extended Witten's proof to include horizons, by proving that the horizon boundary term is zero. We will leave the issue of horizons and tension to future work.

\section{Proof of positivity theorems}

Let $\mathcal{M}$ be a spacetime with $n$ total spacetime dimensions. $\mathcal{M}$ is assumed to have the topology of an $(n-p)$-dimensional noncompact spacetime which is asymptotically flat, cross a $p$-dimensional space which may be compact or noncompact. In the asymptotically flat region, we assume that there is (at least) one spatial-translation Killing vector of the $p$-dimensional space. Further, we assume that in the asymptotic region, the spacetime has a covariantly constant spinor $\psi_{0}$. The spacetime tension $\mu$ will be defined in terms of the long range gravitational field and a spinor field which approaches $\psi_{0}$.

Assume that $\mathcal{M}$ can be foliated by spacelike slices $V_{t}$ with unit timelike normal $n_{a}$ and additionally that it can also be foliated by timelike slices $V_{x}$ with unit spacelike normal $x_{a}$. These surfaces can be used to define coordinates $(t, x)$ by defining the $V_{t}$ and $V_{x}$ to be surfaces of constant $t$ and $x$ respectively. At transverse spatial infinity, we assume that $n^{a}$ and $x^{a}$ are proportional to the asymptotic time and space translation Killing vectors respectively. We will further assume that the spacelike

covariant Lagrangian approach [6] was used to define the tension $\mu$ of a $p$-brane spacetime. It is straightforward to check that these two methods give equivalent results. 
surfaces $V_{t x}$ with both $t$ and $x$ held constant are submanifolds. These foliations allow us to write the metric on $\mathcal{M}$ in the following three forms

$$
\begin{aligned}
g_{a b} & =x_{a} x_{b}+s_{a b} \\
& =-n_{a} n_{b}+h_{a b} \\
& =-n_{a} n_{b}+x_{a} x_{b}+q_{a b} .
\end{aligned}
$$

Here $h_{a b}$ is the Euclidean metric on the hypersurfaces $V_{t}, s_{a b}$ is the Lorentzian metric on the hypersurfaces $V_{x}$, and $q_{a b}$ is the Euclidean metric on the submanifolds $V_{t x}$. The matrices $h_{b}^{a}, s_{b}^{a}$ and $q_{b}^{a}$ then act on vectors as projection operators onto the tangent spaces of the surfaces $V_{t}, V_{x}$ and $V_{t x}$ respectively. In the calculations below, we make use of a frame field $e_{b}^{\hat{a}}$, chosen such that $e^{\hat{t} a}=n^{a}$ and $e^{\hat{x} a}=x^{a}$. Our construction will take place in a volume $\Omega \subset \mathcal{M}$ that is bounded by initial and final spacelike slices $V_{i}$ and $V_{f}$, and by an outer boundary $\partial \Omega_{\infty}$ at infinity in the transverse asymptotically flat directions. We will assume that there are no horizons in $\mathcal{M}$ and correspondingly that the volume $\Omega$ has no inner boundary.

\subsection{A Modification of the Positive Energy Theorem}

We first present a modification of Witten's proof of the positive energy theorem [1] as reformulated by Nester [7]. Our construction gives the purely gravitational contribution to the mass in terms of the metric $q_{a b}$, rather than $h_{a b}$ as in the original proofs. Both $q_{a b}$ and $h_{a b}$ are positive definite, so either construction gives a positivity result, when the spinor field satisfies the Witten-Dirac equation defined in terms of $q_{a b}$ or $h_{a b}$ respectively. The distinction is that $q_{a b}$ is the metric on a co-dimension two slice $V_{x t}$ and $h_{a b}$ is for a co-dimension one slice $V_{t}$. In the case of the spacetime tension $\mu$, it is necessary to reduce by two dimensions to prove positivity. We illustrate the technique first for the mass $M$. This also gives an expression for the mass that is more useful for comparision to the tension.

The Nester 2-form is defined by

$$
B^{a b}=\psi^{\dagger} \gamma^{\hat{t}} \gamma^{a b c} \nabla_{c} \psi
$$

where $\nabla_{a}$ is the covariant derivative operator compatible with the full spacetime metric $g_{a b}$ and $\psi$ is a Dirac spinor. If we define the vector $\xi^{a}=-\psi^{\dagger} \gamma^{\hat{t}} \gamma^{a} \psi$ and require that the spinor field approach a constant spinor $\psi_{0}$ at infinity, then the ADM energymomentum vector $P^{a}$ is given in terms of an integral over the $(n-2)$-dimensional boundary $\left(\partial V_{t}\right)^{\infty}$ at transverse spacelike infinity in a $p$-brane spacetime according to

$$
\begin{aligned}
-P^{a} \xi_{a} & =\frac{1}{16 \pi} \int_{\left(\partial V_{t}\right)^{\infty}} d S_{a}\left(B^{a b}+B^{a b \star}\right) n_{b} \\
& =-\frac{1}{8 \pi} \int_{\left(\partial V_{t}\right)^{\infty}} d S_{a}\left(\psi^{\dagger} h^{a}{ }_{d} h^{b}{ }_{e} \gamma^{d e} \nabla_{b} \psi+\text { c.c. }\right)
\end{aligned}
$$

In particular, if we take the asymptotic value of the spinor $\psi_{0}$ to be an eigenfunction

of $\gamma^{\hat{t}}$, then at infinity $\xi^{a}=(\partial / \partial t)^{a}$ and $P^{a} \xi_{a}=-M$. In order to focus on the 
relationship between the mass and the tension, we will assume such a choice for $\psi_{0}$ below.

The construction proceeds by using Stokes theorem to rewrite the boundary integral (6) as a volume integral. Making use of the identity $\gamma^{a b c} \nabla_{a} \nabla_{c} \psi=-\frac{1}{2} G^{b c} \gamma_{c} \psi$, where $G_{a b}$ is the Einstein tensor and the decomposition of the spatial metric $h_{a b}=$ $q_{a b}+x_{a} x_{b}$, the volume integrand can be written as

$$
\begin{aligned}
n_{b} \nabla_{a} B^{a b}= & -\frac{1}{2} n^{a} G_{a c} \psi \gamma^{\hat{t}} \gamma^{c} \psi+\nabla_{a} \psi^{\dagger} q^{a b} \nabla_{b} \psi-\left(\nabla_{a} \psi^{\dagger} q_{c}^{a} \gamma^{c}\right)\left(q_{d}^{b} \gamma^{d} \nabla_{b} \psi\right) \\
& -x^{a} \nabla_{a} \psi^{\dagger} \gamma^{\hat{x}}\left(\gamma^{b} q_{b}{ }^{c} \nabla_{c} \psi\right)-\left(\nabla_{a} \psi^{\dagger} q^{a}{ }_{b} \gamma^{b}\right) \gamma^{\hat{x}} x^{c} \nabla_{c} \psi
\end{aligned}
$$

Now suppose that in the region $\Omega$, the spinor $\psi$ satisfies a modified Dirac-Witten equation given by

$$
q_{b}^{c} \gamma^{b} \nabla_{c} \psi=0
$$

Then the third, fourth, and fifth terms on the right hand side of (8) are zero. Equation (9) is a differential equation defined on each of the slices $V_{t x}$. So, the solution $\psi$ will depend on the coordinates $x$ and $t$ as parameters. We assume that this dependence is sufficiently smooth to allow us to use Stokes theorem below. If we now integrate equation (8) over any of the hypersurfaces $V_{t}$, then using Einstein's equations $G_{a b}=$ $8 \pi T_{a b}$, the result is

$$
M=\frac{1}{8 \pi} \int_{V_{t}} \sqrt{h}\left(8 \pi T_{a b} n^{a} \xi^{b}+2 \nabla_{a} \psi^{\dagger} q^{a b} \nabla_{b} \psi\right) .
$$

This gives an alternative demonstration that the ADM mass is positive if the stressenergy satisfies the dominant energy condition.

In the original Witten-Nester construction, the metric $h_{a b}$, rather than $q_{a b}$, appears in the first line of equation (8), and the second line of (8) does not arise at all. The spinor field must be a solution to $h_{b}^{c} \gamma^{b} \nabla_{c} \psi=0$. Let us compare the Witten-Nester construction to the present case. We are able to reduce by two dimensions and still prove positivity, because the extra terms generated are still proportional to the Dirac equation (9). It is reasonable to ask if one could reduce further, i.e., let $g_{a b}=L_{a b}+q_{a b}$ where $L_{a b}, q_{a b}$ are orthogonal, and $q_{a b}$ is positive definite. Can the expression for $M$ be written just in terms of the transverse metric $q_{a b}$ ? Starting with the two-form $B^{a b}$ the answer is no, at least with the requirment that $\psi$ satisfy a Dirac equation. Cross terms arise which are not zero or sign definite. It would be interesting to know if starting with a higher dimension form would yield a positiviy proof for the mass per unit length of a general $p$-brane.

\subsection{Positivity theorem for the gravitational component of tension}

We want to ask whether, or not, there is a similar positivity result associated with the tension of a $p$-brane spacetime. We start by defining a Nester form $E^{a b}$ in analogy 
with equation (5), but with $x^{a}$ assuming the special role played by the time direction in the positive energy construction above. Let

$$
E^{a b}=\psi^{\dagger} \gamma^{\hat{x}} \gamma^{a b c} \nabla_{c} \psi
$$

Then it is straightforward to show that

$$
\begin{aligned}
x_{b} \nabla_{a} E^{a b}= & -\frac{1}{2} x^{a} G_{a c} \psi^{\dagger} \gamma^{\hat{x}} \gamma^{c} \psi+\nabla_{a} \psi^{\dagger} q^{a b} \nabla_{b} \psi-\left(\nabla_{a} \psi^{\dagger} q_{c}^{a} \gamma^{c}\right)\left(q_{d}^{b} \gamma^{d} \nabla_{b} \psi\right) \\
& -\nabla_{a} \psi^{\dagger} n^{a} \gamma^{\hat{t}}\left(\gamma^{b} q_{b}{ }^{c} \nabla_{c} \psi\right)-\left(\nabla_{a} \psi^{\dagger} q^{a}{ }_{b} \gamma^{b}\right) \gamma^{\hat{t}} n^{c} \nabla_{c} \psi .
\end{aligned}
$$

If the spinor $\psi$ satisfies the Dirac-Witten equation (9), then again only the first two terms on the right hand side of equation (12) are nonzero. Stokes theorem can then be used to integrate (12) over a Lorentzian slice $V_{x}$. The boundary of this slice $\partial V_{x}$ includes initial and final slices $V_{x}^{i}$ and $V_{x}^{f}$, as well as the boundary at transverse spatial infinity. However, direct computation shows that if $\psi$ solves the Dirac equation, then the quantity $n_{a} x_{b} E^{a b}=0$. Therefore $V_{x}^{i}$ and $V_{x}^{f}$ do not contribute to the tension. The boundary at infinity is a product of a spatial surface that we denote $\left(\partial V_{t x}\right)^{\infty}$ and a finite time interval $\Delta t$ between initial and final time slices. Because the spacetime is transverse asymptotically flat, the integrand at infinity is independent of the time coordinate $t$ and the integral is proportional to $\Delta t$. This leads to an expression for the tension $\mu$ per unit time as the boundary integral

$$
\begin{aligned}
\mu & =\frac{1}{8 \pi(\Delta t)} \int_{\left(\partial V_{x}\right)^{\infty}} d t d a_{b}\left(E^{b c}+E^{b c *}\right) x_{c} \\
& =-\int_{\left(\partial V_{t x}\right)^{\infty}} d a_{a} s_{{ }^{a}} s^{b}{ }_{e}\left(\psi^{\dagger} \gamma^{d e} \nabla_{b} \psi+\text { c.c. }\right) .
\end{aligned}
$$

It turns out that the definition of tension given in equation (13) is the same as previously given, without reference to spinor fields, in [2] and [3]. The argument is a bit detailed, and we defer it until the end of the section.

Using Stokes theorem, then on solutions to the Dirac equation equation (12) becomes

$$
8 \pi \mu=\frac{1}{8 \pi(\Delta t)} \int_{V_{x}} \sqrt{-s}\left(-8 \pi T_{a b} \chi^{a} x^{b}+2 q^{a b} \nabla_{a} \psi^{\dagger} \nabla_{b} \psi\right)
$$

where $\chi^{c}=\frac{1}{2}\left(\psi^{\dagger} \gamma^{\hat{x}} \gamma^{c} \psi+\psi^{\dagger} \gamma^{c \dagger} \gamma^{\hat{x}} \psi\right)$. When $\psi_{0}$ is chosen to be an eigenfunction of $\gamma^{\hat{t}}$, then the vector $\chi^{c}$ approaches $\frac{\partial}{\partial x}$ at infinity. Equation (15) shows that the purely gravitational contribution to the tension, the second term in the integrand, is positive definite.

If the spacetime is static with timelike Killing vector $l^{a}=\frac{\partial}{\partial t}$ everywhere, the volume integral in equation (15) can be reduced to an integral over a spacelike slice $V_{t x}$. Choose a slice $V_{t x}^{i}$, and foliate $\Omega$ with surfaces $V_{t x}$ by moving $V_{t x}^{i}$ along the integral curves of $l$. The volume integrand is independent of the symmetry coordinate $t$, and (15) becomes

$$
8 \pi \mu=\int_{V_{t x}} \sqrt{-s}\left(-8 \pi T_{a b} \chi^{a} x^{b}+2 q^{a b} \nabla_{a} \psi^{\dagger} \nabla_{b} \psi\right)
$$


This relation illustrates why we have called $\mu$ the tension. The stress-energy contributes to $\mu$ as one would expect for a tension. For example, suppose the stress energy is a perfect fluid. Since the spacetime is static, the velocity of the fluid $u^{a}$, is proportional to $l^{a}$. Choose the slices so that $n^{a}=u^{a}$. Then $T_{a b} \chi^{a} x^{b}=\psi^{\dagger} \psi T_{\hat{x} \hat{x}}$, so that the stress energy term in (16) is minus the pressure in the $\hat{x}$-direction, times the positive quantity $\psi^{\dagger} \psi$, which approaches one at infinity. For comparision, in this same example, the stress energy contribution to $M$ is $\psi^{\dagger} \psi T_{\hat{t} \hat{t}}$. Equations (15), (16) constitute our main results.

We can add some intuition to the definition of $\mu$ by recalling a previous result [2]. Let $g_{a b}^{0}$ have the spatial translation Killing field $\left(\frac{\partial}{\partial x}\right)^{b}$ everywhere, and let $g_{a b}$ be perturbatively close to $g_{a b}^{o}$. We showed that for arbitrary perturbations, $\delta \mu=-\int \delta T_{a b} x^{a}\left(\frac{\partial}{\partial x}\right)^{b}$, which is how one expects the stress-energy to contribute to the tension. For comparision, the perturbation to the mass satisfies $\delta M=\int \delta T_{a b} n^{a}\left(\frac{\partial}{\partial t}\right)^{b}$. These results agree with the perturbative limits of the spinor relations (16) and (10) respectively, though some care must be taken in verifying this, since the spinor field receives first order corrections and this changes the associated vector. If the background spacetime $g_{a b}^{0}$ contains a black brane horizon, then both $\delta M$ and $\delta \mu$ receive contributions from the boundary at the horizon. For the mass, this is the First Law, $\delta M=\frac{1}{8 \pi} \kappa \delta A+\int \delta T_{a b} n^{a}\left(\frac{\partial}{\partial t}\right)^{b}$. For the tension, we derived the relation [2]

$$
\delta \mu=\frac{1}{(n-2) 8 \pi} \kappa \delta A-\int \delta T_{a b} x^{a}\left(\frac{\partial}{\partial x}\right)^{b}
$$

where $A$ is the area of the horizon and $\kappa$ the surface gravity. A topic for future work is to investigate the extension of the spinor result (16) to spacetimes with horizons.

Lastly, we return to the demonstration that equation (13) does indeed agree with the definition of the tension $\mu$ given in references [2] and [3]. In the asymptotic region we can write the metric as $g_{a b}=g_{a b}^{0}+\delta g_{a b}$, where the background metric $g_{a b}^{0}$ is flat with periodic identifications in the directions along the brane ${ }^{2}$. Similiarly, $s_{a b}=s_{a b}^{0}+\delta s_{a b}, q_{a b}=q_{a b}^{0}+\delta q_{a b}$. Let $V^{a}$ be the spatial translation Killing field of $g_{a b}^{0}$. The expression for $\mu$ given in reference [2] is

$$
\mu=\frac{1}{16 \pi} \int d a_{b}\left[\tilde{D}_{a}\left(\delta s_{c d} g^{a c} g^{b d}\right)-\tilde{D}^{b}\left(\delta s_{c d} g^{c d}\right)\right] V^{\hat{x}},
$$

where $\tilde{D}$ satisfies $\tilde{D}_{a} s_{b c}^{0}=0$.

The definition (13) must be rewritten in terms of the perturbation to the metric. The boundary integral in equation (13) depends on the derivative operator $\nabla_{a}$. In the asymptotic regime it is useful to write the derivative operator as $\nabla_{a}=\nabla_{a}^{0}+\left(\nabla_{a}-\nabla_{a}^{0}\right)$, where $\nabla_{a}^{0}$ denotes the flat derivative operator of the flat background metric $g_{a b}^{0}$. If we now let $E^{a b}\left[\nabla^{0}\right]$ be the Nester form defined with respect to the flat derivative operator, then the quantity $E^{a b}\left[\nabla^{0}\right] x_{a}$ can be shown to vanish at infinity. In order to evaluate the boundary term in (13), it then suffices to compute the quantity

\footnotetext{
${ }^{2}$ If the compact space is not flat, for example, a Calabi-Yau space cross an $S^{1}$, there are additional terms in equation (23) below.
} 
$b^{a}=q_{b}^{a} E^{b c}\left[\nabla-\nabla^{0}\right] x_{c}$ in terms of the perturbation $\delta g_{a b}$ in the asymptotic regime. The operator $\left(\nabla_{a}-\nabla_{a}^{0}\right)$ acting on a tensor field is given in terms of the connection coefficients

$$
\Gamma_{c e}^{d}=\frac{1}{2} g^{d b}\left(\nabla_{c}^{0} \delta g_{b e}+\nabla_{e}^{0} \delta g_{b c}-\nabla_{b}^{0} \delta g_{c e}\right) .
$$

Making use of this result, we find that

$$
b^{a}+b^{a *}=\frac{1}{4}\left(q_{b}^{a} \psi^{\dagger}\left\{q_{c}^{d}\left(\gamma^{b c} \gamma^{m n}-\gamma^{m n \dagger} \gamma^{b c}\right)+n^{d} \gamma^{\hat{t}}\left(-\gamma^{b} \gamma^{m n}+\gamma^{m n} \gamma^{b}\right)\right\} \psi\right) \nabla_{[m} \delta g_{n] d}
$$

In order to proceed further we must specify the rates at which the different components of $\delta g_{a b}$ vanish at transverse spatial infinity. Letting $i, j \neq x, t$, we assume that the components $\delta g_{t t}, \delta g_{x x}$ and $\delta g_{i j}$ fall off as $1 / r^{n-p-3}$ and that the components $\delta g_{t i}$, $\delta g_{t x}$ and $\delta g_{x i}$ fall off at a faster rate. These latter conditions imply, in particular, that the extrinsic curvatures of the slices $V_{t}$ and $V_{x}$ fall off faster than $1 / r^{n-p-3}$. Calculations then lead to useful intermediate results. In particular, it follows that

$$
q^{a m} n^{e} \gamma^{n} \nabla_{m} \delta g_{n e} \simeq q^{a m} \nabla_{m} \gamma^{\hat{t}} \delta g_{\hat{t} \hat{t}}
$$

where the equality holds up to terms which vanish in the limit that the boundary integral goes to infinity. Similarly, it follows that

$$
q_{c}^{e} \gamma^{m n} \nabla_{[m} \delta g_{n] e} \simeq q_{c}^{e} \gamma^{m n} D_{[m} \delta q_{n] e}
$$

where $D_{a}$ is the derivative operator on the surfaces $V_{t x}$ satisfying $D_{a} q_{b c}=0$. Putting these results together allows one to rewrite the tension boundary term in equation (13) as

$$
\mu=\frac{1}{16 \pi} \int d a_{b}\left[D_{a}\left(\delta q_{c d} g^{a c} g^{b d}\right)-D^{b}\left(\delta q_{c d} g^{c d}\right)+D^{b} \delta g_{\hat{t} \hat{t}}\right] \psi_{0}^{\dagger} \psi_{0}
$$

Use $q_{b}^{a} D_{a} \delta q_{c d} \simeq q_{b}^{a} \tilde{D}_{a}\left(\delta s_{m n} q_{a}^{m} q_{b}^{n}\right)$ and $\delta s_{c d} g^{c d}=\delta q_{c d} g^{c d}-\delta g_{\hat{t} \hat{t}}$. Then the boundary term in equation (18), given in terms of $\delta s_{a b}$, agrees with equation (23) with the identification $V^{\hat{x}}=\psi_{0}^{\dagger} \psi_{0}$.

\subsection{Comparing Mass and Tension}

One notices an interesting feature when comparing the spinor expressions for the mass and tension in static spacetimes. Equations (10) and (16) show that the purely gravitational contribution to the mass and the tension are the same when the volume elements are equal. In such a case the difference between the mass and the tension is only due to the stress energy terms. One situation where this occurs, noted in [3], is when $\frac{\partial}{\partial x}$ is a Killing vector throughout the spacetime, and the spacetime is (globally) boost-invariant in the $\hat{x}-\hat{t}$ plane. Then the symmetry implies that the boundary terms for $M$ and $\mu$ are the same. To see that there are less restrictive cases, choose the timelike normal to be parallel to the static Killing field, $n_{a}=-N \nabla_{a} t$. The volume elements are equal if $x_{a}$ can be chosen such that $x_{a}=N \nabla_{a} x$, for example, if 
the metric can be put in the form $d s^{2}=N^{2}\left(x, x^{j}\right)\left(-d t^{2}+d x^{2}\right)+q_{i j} d x^{i} d x^{j}$. That is, $\frac{\partial}{\partial x}$ need not be a Killing vector throughout the spacetime, but there is a local boost invariance in the $\hat{x}-\hat{t}$ plane. Physically, one would expect this to result from a source with $p_{x}=-\rho$ locally, but that the pressure depends on position along the string.

A related issue is whether, or not, there are solutions which are static but not translationally invariant. An example of such a solution has been conjectured in analysis of the Gregory-Laflamme instability of the black string [9]. It is conjectured that (a) the unstable horizon evolves to a static, translationally non-invariant black string [10] [11], and that (b) at the onset of instability there is also a static translationally non-invariant state [12] [13]. Our present results do not apply to the black string since we have not included horizons. So, as far as the above spinor expressions apply, we are interested in smooth fill-ins of the conjectured solutions (a), (b). This leads to an interesting question. An $x$-dependent pressure means that there is a pressure gradient along the brane, which usually makes things flow, and would contradict the assumption of staticity. On the other hand, a star has a radial pressure gradient. The star can still be static because gravity is attractive. So it may be that the positive tension from the gravitational field can balance a pressure gradient. Then one can understand the positivity of the gravitational tension as a reflection of the attractiveness of gravity. Perhaps the non-symmetric static black-string solutions are limiting cases of this sort of equilibrium.

Acknowledgements: I would like to thank David Kastor and Subir Mukhopadhyay for useful conversations, and the Kavli Institute for Theoretical Physics and the Aspen Center for Physics for their hospitality. This work was supported in part by NSF grant PHY-0244801.

\section{References}

[1] E. Witten, "A Simple Proof Of The Positive Energy Theorem," Commun. Math. Phys. 80, 381 (1981).

[2] J. Traschen and D. Fox, "Tension perturbations of black brane spacetimes," arXiv:gr-qc/0103106.

[3] P. K. Townsend and M. Zamaklar, "The first law of black brane mechanics," Class. Quant. Grav. 18, 5269 (2001) [arXiv:hep-th/0107228].

[4] T. Regge and C. Teitelboim, "Role Of Surface Integrals In The Hamiltonian Formulation Of General Relativity," Annals Phys. 88, 286 (1974).

[5] C. Teitelboim, "Surface Integrals as Symmetry Generators in Supergravity Theory", Physics Letters 69B, 240 (1977).

[6] L. F. Abbott and S. Deser, "Stability Of Gravity With A Cosmological Constant," Nucl. Phys. B 195, 76 (1982). 
[7] J. Nester, "A New Gravitational Expression with a Simple Positivity Proof," Phys. Lett. 83A, 241 (1981).

[8] G. W. Gibbons, S. W. Hawking, G. T. Horowitz and M. J. Perry, "Positive Mass Theorems For Black Holes," Commun. Math. Phys. 88, 295 (1983).

[9] R. Gregory and R. Laflamme, "Black Strings And P-Branes Are Unstable," Phys. Rev. Lett. 70, 2837 (1993) [arXiv:hep-th/9301052].

[10] G. T. Horowitz and K. Maeda, "Fate of the black string instability," Phys. Rev. Lett. 87, 131301 (2001) [arXiv:hep-th/0105111].

[11] G. T. Horowitz and K. Maeda, "Inhomogeneous near-extremal black branes," Phys. Rev. D 65, 104028 (2002) [arXiv:hep-th/0201241].

[12] S. S. Gubser, "On non-uniform black branes," Class. Quant. Grav. 19, 4825 (2002) [arXiv:hep-th/0110193].

[13] H. S. Reall, "Classical and thermodynamic stability of black branes," Phys. Rev. D 64, 044005 (2001) [arXiv:hep-th/0104071]. 